\title{
Fatores de risco associados ao comportamento alimentar inadequado em futebolistas
}

CDD. 20.ed. 796.027

796.33

\author{
Leonardo de Sousa FORTES \\ Marcelo de Oliveira MATTA* \\ Santiago Tavares PAES* \\ Maria Elisa Caputo FERREIRA*
}

*Faculdade de EducacãoFísicae Desportos, Universidade Federal de Juiz de Fora

\begin{abstract}
Resumo
0 comportamento alimentar inadequado (CAI) parece estar associado a diversos fatores. No entanto, a população de atletas, principalmente do sexo masculino, é carente de investigações deste tipo. 0 objetivo do presente estudo foi identificar fatores de risco para o CAl em jovens futebolistas. Foram avaliados 271 futebolistas de Juiz de Fora/MG. Avaliou-se o CAI mediante aplicação do Eating Attitudes Test (EAT-26). A insatisfação corporal foi verificada por intermédio do Body Shape Questionnaire. Aferiu-se massa corporal e estatura para calcular-se o índice de massa corporal, além de dobras cutâneas com o propósito de estimar a adiposidade corporal. Conduziu-se regressão logística binária para avaliar riscos sobre o CAl, incutindo nivel de significância de 5\%. Observou-se que somente a insatisfação corporal manteve-se associada ao CAl, tanto no modelo simples, quanto no ajustado para todas as variáveis ( $\mathrm{p}<$ $0,05)$. Concluiu-se que a insatisfação corporal apresentou riscos para a manifestação do CAl.
\end{abstract}

UNITERMOS: Transtornos alimentares; Atletas; Futebol.

\section{Introdução}

O esporte infanto-juvenil apresenta várias peculiaridades em seu cotidiano. Dentre elas, encontram-se a participação em competições, assim como a exigência da otimização do rendimento atlético. Portanto, é preciso que o atleta desenvolva suas capacidades funcionais (potência aeróbia, força explosiva, resistência anaeróbia, etc), biomecânicas (técnica) e psicológicas (concentração, tomada de decisão, motivação, etc), caso queira elevar o nível de desempenho. Em praticamente todas as modalidades esportivas parece notória a exigência destas características, como é o caso do futebol.

O futebol é uma das modalidades mais praticadas no mundo, apresentando múltiplos fatores determinantes para o desenvolvimento de atletas (RocHA, Bartholo, Melo \& Soares, 2011). Além de características antropométricas, fisiológicas e biomecânicas, as psicológicas também apresentam importância fundamental no processo de formação esportiva, como éo caso das variáveis comportamentais (DENOMA, SCARINGI, GORdON, VAN ORDEN \& JoINER, 2009; ForTeS \& Ferreira, 2011; Torstveit \& Sundgot-Borgen,
2005; Vieira, Vieira, Amorim \& Rocha, 2009). Alguns atletas utilizam métodos compensatórios como estratégias para controle de peso (BAUM, 2006; FILAIRE, Rouveix, Pannafieux \& Ferrand, 2007; Fortes, Miranda, Amaral \& Ferreira, 2011). Pode-se citar: indução de vômitos, restrição alimentar e atividade física extenuante. Estas atitudes podem ser prejudiciais a saúde do jovem, além de repercutir negativamente em seu rendimento esportivo (Fortes, Paes, Amaral \& Ferreira, 2012; Perini, Vieira, Vigário, Oliveira, Ornelas \& Oliveira, 2009; Vieira et al., 2009).

O comportamento alimentar inadequado, juntamente com a insatisfação com peso e aparência física podem desencadear síndromes psicológicas (FORTES et al., 2012; PeriNI et al., 2009). A adiposidade corporal elevada e a obesidade também têm sido associadas ao desenvolvimento de psicopatologias (VIEIRA et al., 2009). Além destas, acredita-se que o processo maturacional possa exercer influência sobre variáveis comportamentais, podendo ser considerado fator de risco para a instalação dos transtornos alimentares 
(TAs). A anorexia nervosa e bulimia nervosa são os TAs mais comumente encontrados na população geral (Schaal, Tafflet, Nassif, Thibault, Pichard, Alcotte, Guillet, Berthelot, Simon \& Toussaint, 2011), apresentando prevalências de $1 \%$ e $4 \%$, respectivamente (PERINI et al., 2009). No entanto, parece que no âmbito esportivo suas ocorrências são maiores, pois alguns autores têm identificado altas prevalências de comportamentos alimentares inadequados entre atletas (BAUM, 2006; FILAIRE et al., 2007; SCHAAL et al., 2011; Torstveit \& Sundgot-Borgen, 2005).

Segundo Torstveit e Sundgot-Borgen (2005), o ambiente competitivo pode ser considerado agente potencializador para o surgimento de tais doenças. Estudos têm apresentado que a pressão imposta por treinadores, familiares e patrocinadores no anseio

\section{Método}

\section{Participantes}

Trata-se de um estudo transversal realizado no ano de 2011 com futebolistas de 10 a 19 anos de idade, do sexo masculino, pertencentes a cinco clubes da cidade de Juiz de Fora/MG, regularmente registrados na Liga de Futebol de Juiz de Fora.

A população de atletas deste estudo foi estimada em 658, segundo informações da Liga de Futebol da referida cidade. Para o cálculo da amostra, adotou-se percentual máximo de $20 \%$ para comportamento alimentar, utilizando a equação de FIELD (2009), que considera erro relativo tolerável de cinco pontos percentuais e intervalo de confiança de 95\%, obtendo-se um tamanho amostral estimado de 180 adolescentes.

Para participar do estudo, os sujeitos deveriam apresentar o termo de consentimento livre e esclarecido (TCLE) assinado pelo responsável (caso menor de 18 anos), treinar sistematicamente futebol pelo menos três vezes na semana com duração mínima de uma hora por sessão, além de ter participado de alguma competição no ano de 2011.

Desta forma, foram avaliados 302 indivíduos. No entanto, 31 atletas foram excluídos da pesquisa por não responderem os questionários em sua totalidade ou não participarem das aferiçôes antropométricas. Portanto, a amostra final do estudo foi constituída por 271 jovens. por melhores resultados (DENOMA et al., 2009), vestimentas que salientam a forma corporal (FORTES et al., 2012; Krentz \& WARCHSBURGER, 2011) e a ênfase atribuída a magreza são fatores de risco que podem predispor atletas a inadequação alimentar (BAum, 2006; Perini et al., 2009). Entretanto, pesquisas têm sido desenvolvidas somente utilizando o sexo feminino, ou seja, o campo acadêmico não tem dado devida atenção ao público masculino. Além do mais, até o momento, não foi encontrada nenhuma investigação avaliando fatores de risco para o comportamento alimentar inadequado com futebolistas adolescentes. Tomando isto por base, o objetivo do presente estudo foi identificar fatores de risco para o comportamento alimentar inadequado em atletas adolescentes de futebol.

\section{Instrumentos}

\section{Comportamento alimentar inadequado}

Foi avaliado mediante aplicação do Eating Attitudes Test em sua versão com 26 questões (EAT-26). Este instrumento busca avaliar dieta, comportamentos bulímicos, restrição alimentar, preocupação com os alimentos e autocontrole oral. O EAT-26 apresenta pontuações em escala do tipo Likert (Nunca, Quase Nunca e Poucas Vezes $=0$; Às vezes $=1$; Muitas Vezes $=2$; Sempre $=3$ - exceção da questão 25 que a pontuação é invertida) variando de zero a 78 . O "score" igual ou superior a 20 pontos indica os indivíduos supostamente suscetíveis ao desenvolvimento de distúrbios de conduta alimentar, além da presença de padrões alimentares anormais, não revelando, contudo, a possível psicopatologia subjacente ao comportamento manifesto. A versão utilizada no presente estudo foi a de Fortes, Amaral, Conti, CORDÁs e FERREIRA (no prelo) que apresentou valor alpha de Cronbach de 0,88 e não identificou diferença entre o teste-reteste. Para a presente amostra, calculouse a consistência interna, obtendo-se valor de 0,90 .

\section{Insatisfação corporal}

Avaliou-se por intermédio do Body Shape Questionnaire (BSQ). Trata-se de um questionário 
auto-reportado constituído por 34 perguntas que se propõem avaliar a preocupação com peso e forma física. O BSQ é uma escala do tipo Likert com seis opçōes de resposta para cada questão (Nunca $=1$ até Sempre =6). Ele classifica níveis de insatisfação a respeito do corpo, sendo: $<80$ pontos livre de insatisfação corporal, entre 80 e 110 leve insatisfação, entre 110 e 140 insatisfação moderada e pontuações acima de 140 grave insatisfação corporal, ou seja, quanto maior o escore, maior a insatisfação com o corpo. A versão utilizada foi validada para adolescentes brasileiros (CONTI, CORDÁs \& LATORRE, 2009) e sua análise de consistência interna revelou alpha de 0,96 e coeficiente de correlação entre os escores do teste-reteste significativo. Calculouse a consistência interna para a presente amostra, identificando-se valor alpha de 0,92.

\section{Maturação somática}

O pico de velocidade de crescimento em altura (PVA) é o indicador de maturidade mais comumente usado para se avaliar a maturação somática, porém são necessários dados longitudinais, o que demanda tempo e custo financeiro altíssimo. Por isso, Mirwald, Baxter Jones, Bailey e Beunen (2002) desenvolveram uma fórmula por meio de análise de regressão múltipla utilizando o banco de dados de dois estudos longitudinais para estimar a idade de PVA. Para isso, é necessário aferir uma única vez: estatura, peso e altura tronco-cefálica. O comprimento de pernas é obtido pela diferença entre estatura e altura tronco-cefálica. Basta combinar estas variáveis com a idade cronológica na seguinte fórmula:

\section{Meninos:}

Maturidade somática $=-9,236+0,0002708 x$ interação do comprimento de pernas e altura sentado - 0,001663 x interação entre idade e comprimento de pernas $+0,007216 \mathrm{x}$ interação entre idade e altura sentado $+0,02292 \times$ razão entre peso por altura.

A fórmula fornece resultados com pontuaçôes negativas e positivas. Valor negativo significa que o sujeito ainda vai atingir o PVA e valor positivo apresenta que o indivíduo já passou do PVA. Segundo Machado, Bonfim e Costa (2009), a classificação pode ser feita da seguinte maneira:

$<-1$ = Pré Estirão de Crescimento em Estatura Entre -1 e $+1=$ Durante Estirão de Crescimento em Estatura

$>+1=$ Pós Estirão de Crescimento em Estatura

\section{Antropometria}

Foram coletadas massa corporal, estatura e dobras cutâneas (subescapular e triciptal). Utilizaram-se os procedimentos descritos pela ISAK (2001). A massa corporal e a estatura foram mensuradas com os avaliados descalços e com o mínimo de roupa. Utilizou-se uma balança eletrônica portátil da marca Tanita, com precisão de $0,1 \mathrm{~kg}$, para o peso, e um estadiômetro portátil, com precisão de $0,1 \mathrm{~cm}$, da marca Tonelli, para a estatura. Essas variáveis foram mensuradas uma única vez. O Índice de Massa Corporal (IMC) foi obtido pela razão entre massa corporal $(\mathrm{kg})$ dividido pelo quadrado da estatura $(\mathrm{m})$.

A classificação do estado nutricional foi obtida mediante os pontos de corte do IMC estabelecidos pela World Health Organization (2007), que os separa por intermédio do percentil $(5,85$ e 95$)$ em quatro grupos contrastes segundo sexo e idade cronológica: baixo peso (percentil $\leq 5$ ), eutrófico (percentil $>5 \mathrm{e}<85$ ), sobrepeso (percentil $\geq 85 \mathrm{e}<$ 95) e obeso (percentil $\geq 95$ ).

As medidas das dobras cutâneas foram efetuadas em triplicata, de forma não consecutiva. Estas foram mensuradas com plicômetro da marca Lange com a precisão de $0,1 \mathrm{~mm}$. Estas medidas foram realizadas do lado direito do corpo. A medição da dobra cutânea do tríceps foi feita na parte posterior do braço, no ponto médio entre o processo acromial e o olécrano. A medição da dobra cutânea subescapular foi realizada $2 \mathrm{~cm}$ abaixo do ângulo inferior da escapula a uma inclinação de $45^{\circ}$ em relação ao lado do corpo.

Para os cálculos da percentagem de gordura $(\% \mathrm{G})$, utilizou-se a equação de predição proposta por Slaughter, Lohman, Boileau, Hoswill, Stillman e Yanloan (1988). Determinou-se a adiposidade corporal pelo percentual de gordura. A classificação dessa variável foi atribuída pelos pontos de corte estabelecidos por LOHMAN (1987), que levam em consideração o sexo do sujeito.

\section{Procedimentos}

Os procedimentos foram realizados no horário do treinamento das equipes em dois dias subsequentes. Todos os clubes disponibilizaram salas e ambientes para proceder tais avaliaçôes.

As coletas de questionários foram realizadas por apenas um pesquisador (LSF). Este ficou responsável pela aplicação destes instrumentos no primeiro momento. O segundo encontro destinou-se as aferições antropométricas (peso, estatura, altura 
tronco-cefálica e dobras cutâneas), cuja realização ficou restrita a outro pesquisador (MOM) com experiência neste tipo de avaliação.

No primeiro encontro aplicou-se os instrumentos BSQ e EAT-26. Os questionários foram entregues aos atletas que receberam a mesma orientação verbal. A orientação escrita dos procedimentos adequados constava nos mesmos. Eventuais dúvidas foram esclarecidas pelos responsáveis pela aplicação destes instrumentos. Os sujeitos do estudo não comunicavam entre si. Efetuou-se a distribuição dos questionários no momento que os atletas adentravam no ambiente (sala) e o preenchimento destes constituiu-se de caráter voluntário. Não houve limite de tempo para preenchê-los.

No segundo momento os atletas foram conduzidos para aferição das variáveis antropométricas. A entrada dos atletas na sala de avaliação foi individualizada, permitindo-se outro sujeito adentrar no ambiente somente após seu colega de equipe retira-se do recinto.

Todos os procedimentos somente foram realizados após aprovação do projeto no Comitê de Ética e Pesquisa em Seres Humanos da Universidade
Federal de Juiz de Fora, recebendo parecer número 232/2010, de acordo com a resolução 196 de 1996.

\section{Análise dos dados}

Utilizou-se medidas de tendência central (média e desvio padrão) para descrever as variáveis insatisfação corporal e comportamento alimentar. Realizou-se análise univariada de covariância (ANCOVA), utilizando idade, percentual de gordura e IMC como covariáveis, para comparar o comportamento alimentar entre classificaçōes de maturação somática, adiposidade corporal e estado nutricional. Conduziu-se regressão logística binária para avaliar riscos ("odds ratio") do estado nutricional, adiposidade corporal e insatisfação corporal sobre o comportamento alimentar. Entretanto, foi necessário agrupar as classificações de insatisfação corporal do BSQ (leve, moderada e grave) em “insatisfeitos”. Além disso, considerou-se somente três grupos de adiposidade corporal para esta análise: baixo (baixo e muito baixo), normal (normal) e alto (alto e muito alto). Os testes estatísticos foram realizados no "software" SPSS versão 17.0, incutindo nível de significância de 5\%.

\section{Resultados}

Participaram do estudo 271 atletas de futebol com as seguintes características: idade de 14,76 ( \pm $1,66)$ anos, $20,86( \pm 2,76) \mathrm{kg} / \mathrm{m}^{2}$ de IMC e percentual de gordura de $17,78( \pm 6,69)$.
A TABELA 1 apresenta média e desvio padrão do EAT-26 entre diferentes classificações de maturação somática, adiposidade corporal e estado nutricional. Não foram observadas diferenças estatisticamente significativas de comportamento alimentar entre os grupos.

TABELA 1 - Comparação do comportamento alimentar inadequado entre diferentes classificações de maturação somática, adiposidade corporal e estado nutricional de futebolistas adolescentes.

\begin{tabular}{lcc}
\hline Classificação & EAT-26 \\
\hline & Maturação somática & \\
Pré Estirão & & $10,95( \pm 3,01)$ \\
Estirão & & $12,25( \pm 1,21)$ \\
Pós Estirão & Adiposidade corporal & $13,72( \pm 1,90)$ \\
& & $14,34( \pm 2,26)$ \\
Baixo & & $14,80( \pm 1,27)$ \\
Normal & & $12,88( \pm 1,59)$ \\
Alto & Estado nutricional & \\
Baixo Peso & & $10,12( \pm 4,14)$ \\
Normal & & $12,51( \pm 0,86)$ \\
Sobrepeso & & $12,60( \pm 3,00)$ \\
Obeso & & $15,78( \pm 4,95)$ \\
\hline
\end{tabular}


Em relação a razão de chances ("odds ratio") avaliada por meio da regressão logística binária, observou-se que somente a insatisfação corporal manteve-se associada ao comportamento alimentar inadequado, tanto no modelo simples, quanto no ajustado para todas as variáveis $(\mathrm{p}<0,05)$.

TABELA 2 - Razão de chances para comportamento alimentar inadequado (categoria de referência: negativo) em futebolistas adolescentes segundo insatisfação corporal, estado nutricional e adiposidade corporal.

\begin{tabular}{llllllll}
\hline Variável & Classificação & OR & IC (95\%) & p valor & OR* $^{*}$ & IC (95\%) & p valor \\
\hline IC & Satisfeito & 1,00 & & & 1,00 & & \\
& Insatisfeito & 4,48 & $2,17-9,26$ & $\mathrm{p}<0,01$ & 4,60 & $2,01-10,52$ & $\mathrm{p}<0,05$ \\
EN & Baixo Peso & 0,48 & $0,06-3,86$ & & 0,67 & $0,08-5,56$ & \\
& Peso Normal & 1,00 & & & 1,00 & & \\
& Sobrepeso & 1,51 & $0,57-4,03$ & $\mathrm{p}<0,10$ & 0,72 & $0,22-2,31$ & $\mathrm{p}<0,28$ \\
\multirow{2}{*}{$\% \mathrm{G}$} & Obeso & 3,03 & $0,84-10,92$ & & 1,91 & $0,45-8,22$ & \\
& Baixo & 0,35 & $0,11-1,08$ & & 0,39 & $0,13-1,24$ & \\
& Normal & 1,00 & & $\mathrm{p}<0,08$ & 1,00 & & $\mathrm{p}<0,58$ \\
& Alto & 0,85 & $0,43-1,66$ & & 0,66 & $0,31-1,41$ & \\
\hline
\end{tabular}

IC = Insatisfação corporal; $\mathrm{EM}=$ Estado nutricional $\% \mathrm{G}=$ Percentual de gordura; OR=Razão de chances ("odds ratio"); $\mathrm{OR}^{\star}=$ ORajustado para todas as variáveis; IC(95\%) = 95\% de Intervalo de confiança

\section{Discussão}

O presente estudo teve como premissa avaliar fatores de risco associados ao comportamento alimentar inadequado de jovens futebolistas do sexo masculino. A maioria dos estudos publicados até o momento, tem se destinado avaliar fatores de risco relacionados a variáveis comportamentais e afetivas somente entre escolares (Martins, Pelegrini, Matheus \& Petroski, 2010; Petroski, Pelegrini \& Glaner, 2009; Scherer, Martin, Pelegrini, Matheus \& Petroski, 2010). Além disso, foram encontrados poucos estudos avaliando o sexo masculino. Ademais, pesquisadores não têm dado a devida importância destas avaliações ao público de atletas.

A presente pesquisa evidenciou resultados interessantes. Não identificaram-se diferenças de comportamento alimentar entre diferentes classificações de maturação somática, adiposidade corporal e estado nutricional. Talvez estas variáveis não sejam fatores que influenciem negativamente o comportamento alimentar de futebolistas adolescentes. Entretanto, identificaram-se tendências nos resultados. Percebeuse que tanto na maturação somática, quanto no estado nutricional, as pontuações do EAT-26 aumentaram de acordo com suas classificaçôes. Apesar da não significância estatística, parece que os atletas com sobrepeso/obesidade apresentaram maior tendência de inadequação alimentar, assim como é descrito na população de escolares desta faixa etária (MARTins et al., 2010; Scherer et al., 2010). Por outro lado, esperava-se que o avanço maturacional proporcionasse menores riscos de alimentação desordenada entre atletas do sexo masculino. Segundo Malina, BouCHARD e BAR-OR (2009), a gordura corporal diminui e a massa livre de gordura aumenta no decorrer do processo maturacional, o que culturalmente é bem aceito na sociedade e desejado entre estes jovens (BAum, 2006; Filaire et al., 2007; Martins et al., 2010). No entanto, averiguou-se o contrário, meninos que já haviam passado pelo PVA apresentaram maior tendência de comportamento alimentar inadequado, mesmo não tendo sido encontrada diferenças significativas sobre os demais grupos.

A respeito da associação de fatores de risco sobre a variável de desfecho do presente estudo, avaliada por meio da análise de regressão logística, alguns achados merecem ser destacados. No modelo ajustado, somente a insatisfação corporal manteve-se associada ao comportamento alimentar. Identificou-se que atletas insatisfeitos com o corpo apresentaram mais chances $(4,60)$ de inadequação alimentar do que os esportistas satisfeitos. Estes resultados corroboram com achados de outros autores (FILAIRE et al., 2007; Torstveit \& Sundgot-Borgen, 2005). Parece que a preocupação como peso e aparência física também pode ser preditora de hábitos alimentares não saudáveis entre os futebolistas. Entretanto, estimava-se que adiposidade corporal elevada e estado nutricional inadequado também pudessem 
ser fatores de risco para o comportamento alimentar não saudável nesta amostra. Talvez características morfológicas não afetem hábitos alimentares anormais nos atletas adolescentes de futebol. Alguns autores afirmam que jovens esportistas preocupam-se mais com o rendimento atlético do que aspectos da aparência corporal (DENOMA et al., 2009; FoRTES \& FErreira, 2011; ForTes et al., 2011; SchaAl et al., 2011). Por outro lado, o âmbito competitivo parece ter peculiaridades em seu cotidiano que podem predispor com maior facilidade atletas adquirirem comportamentos compensatórios para controle da massa corporal. Pesquisadores têm estimado que pressōes exercidas por treinadores cobrando melhores resultados, gordura corporal inversamente associada ao rendimento esportivo, experiências de alto nível competitivo, além de uniformes que salientam o formato corporal, possam ser fatores considerados de risco para o desencadeamento de TAs (Filaire et al., 2007; Fortes et al., 2012; PERINI et al., 2009; TorstVeit \& Sundgot-Borgen, 2005).

$\mathrm{O}$ tema de fatores de risco para predisposição de alimentação inadequada entre o público de atletas ainda permanece como tópico a ser investigado. A presente pesquisa procurou preencher uma pequena parcela da lacuna do conhecimento existente neste público. No entanto, o presente estudo apresentou importantes limitações. Uma delas foi utilizar como instrumento principal um questionário. Pesquisadores afirmam que os indivíduos podem não responder com fidedignidade a instrumentos autoaplicáveis (SCHAAL et al., 2011; Torstvert \& SundGOT-Borgen, 2005). Portanto, os resultados podem não refletir a realidade do contexto avaliado, visto que o resultado final é fruto de respostas subjetivas. Além disso, utilizou-se método duplamente indireto para calcular o percentual de gordura. Métodos como a Densitometria Radiológica de Dupla Energia (DEXA) e a Bioimpedância elétrica (BIAS) são mais recomendáveis e apresentam menor erro de estimativa desta variável (MALINA, BOUCHARD \& BAR-OR, 2009). Entretanto, estes métodos dependem de alto dispêndio financeiro, além de recursos humanos especializados. Por isso, pesquisadores recomendam para pesquisas com grande número amostral, que se utilize método de fácil e rápida aplicação para estimar o percentual de gordura do avaliado, como é o caso das dobras cutâneas (PerINI et al., 2009; SCHAAL et al., 2011). Estima-se que este seja o primeiro estudo no Brasil a avaliar fatores de risco para variável comportamental em atletas adolescentes masculinos. Ademais, acredita-se que a presente pesquisa mostre resultados importantes que merecem ser discutidos na literatura científica.

Os resultados do presente estudo permitem concluir que somente a insatisfação corporal apresentou riscos para a manifestação do comportamento alimentar inadequado em atletas adolescentes de futebol da cidade de Juiz de Fora/MG. Deste modo, faz-se necessário a implantação de programas de terapia psicológica com estes jovens, visando reduzir a preocupação que os mesmos possuem com peso e aparência física. Acrescentando, supõe-se que menores frequências de cobranças advinda de treinadores para a otimização do desempenho esportivo possam diminuir os sentimentos negativos a respeito do corpo em futebolistas adolescentes.

Sugere-se a realização de estudos com as mesmas características da presente pesquisa que avaliem atletas de outras modalidades esportivas e incluam o sexo feminino nas análises.

\section{Abstract}

Risk factors associated with feeding behavior of players

The inappropriate eating behavior (IEB) appears to be associated with numerous factors. However, the population of athletes, especially males, is lacking in such investigations. The purpose of this study was to identify risk factors for IEB in young footballers. 271 footballers of Juiz de Fora/MG were evaluated. IEB was assessed by applying the Eating Attitudes Test (EAT-26). The body dissatisfaction was verified through the Body Shape Questionnaire. We measured from this weight and height to calculate the body mass index (BMI), and skinfold thickness in order to estimate the adiposity. We carried out binary logistic regression to assess risks on the IEB, instilling a significance level of 5\%. It was observed that only body dissatisfaction remained associated with IEB, both the simple model, as adjusted for all the variables ( $p$ $<0.05$ ). It was concluded that body dissatisfaction presented risks to the manifestation of IEB.

UnITERMs: Eating disorders; Athletes; Soccer. 


\section{Resumen}

Los factores de riesgo asociados con la conducta de alimentación inadecuada en los jugadores de fútbol

El comportamiento inadecuado de alimentos (CIA), parece estar relacionado con varios factores. Sin embargo, la población de atletas, sobre todo varones, está en la necesidad de tales investigaciones. El objetivo de este estudio fue identificar los factores de riesgo para la CIA en los jóvenes futbolistas. Se evaluaron 271 futbolistas de Juiz de Fora/MG. Se evaluó la CIA aplicando el Eating Attitudes Test (EAT26). La insatisfacción corporal se evaluó a través del Body Shape Questionnaire. Se ha medido el peso y la altura para calcular el índice de masa corporal y grosor del pliegue cutáneo con el fin de estimar la grasa corporal. Hemos llevado a cabo una regresión logística binaria para evaluar los riesgos en el CIA, infundiendo un nivel de significación del $5 \%$. Se observó que la insatisfacción corporal sólo se mantuvo asociada con el CIA, tanto en el modelo simple, como el conjunto de todas las variables $(p<0,05)$. Se concluyó que los riesgos de insatisfacción del cuerpo presentes en la manifestación de la CIA.

Palabras Clave: Trastornos de la alimentación; Los atletas; El fútbol.

\section{Referências}

BAUM, A. Eating disorders in the male athlete. Sports Medicine, Auckland, v.36, n.1, p.1-6, 2006.

CONTI, M.A.; CORDÁS, T.A.; LATORRE, M.R.D.O. Estudo de validade e confiabilidade da versão brasileira do body shape questionnaire (bsq) para adolescentes. Revista Brasileira de Saúde Materna e Infantil, Recife, v.9, n.3, p.331-8, 2009. DENOMA, J.M.H.; SCARINGI, V.; GORDON, K.H.; VAN ORDEN, K.A.; JOINER, T.E. Eating disorder symptoms among undergraduate varsity athletes: club athletes, independent exercisers and nonoexercises. International Journal of Eating Disorders, Hoboken, v.12, n.1, p.47-53, 2009.

FIELD, A. Descobrindo a estatística usando o SPSS. Porto Alegre: Artmed, 2009.

FILAIRE, E.; ROUVEIX, M.; PANNAFIEUX, C.; FERRAND, C. Eating atitudes, perfectionism and body-esteem of elite male judoists and cyclists. Journal of Sports Science and Medicine, Bursa, v.6, n.1, p.50-7, 2007.

FORTES, L.S.; AMARAL, A.C.S.; CONTI, M.A.; CORDÁS, T.A.; FERREIRA, M.E.C. Qualidades psicométricas do Eating Attitudes Test (EAT-26) para a população adolescente masculina brasileira. Psicologia: Reflexão e Crítica, Porto Alegre. No prelo. FORTES, L.S.; FERREIRA, M.E.C. Comparação da insatisfação corporal e do comportamento alimentar inadequado em atletas adolescentes de diferentes modalidades esportivas. Revista Brasileira de Educaçáo Física e Esporte, São Paulo, v.25, n.4, p.707-16, 2011. FORTES, L.S.; MIRANDA, V.P.N.; AMARAL, A.C.S.; FERREIRA, M.E.C. Insatisfação corporal de adolescentes atletas e não atletas. Jornal Brasileiro de Psiquiatria, Rio de Janeiro, v.60, n.4, p.309-14, 2011.

FORTES, L.S.; PAES, S.T.; AMARAL, A.C.S.; FERREIRA, M.E.C. Insatisfação corporal e comportamento alimentar inadequado em jovens nadadores segundo níveis econômicos e competitivos. Jornal Brasileiro de Psiquiatria, Rio de Janeiro, v.61, n.1, p.20-4, 2012.

INTERNATIONAL SOCIETY FOR ADVANCEMENT FOR KINEANTHROPOMETRY (ISAK). International standards for anthropometric assessment. 1st ed. Sidney: National Library of Australia, 2001.

KRENTZ, E.M.; WARSCHBURGER, P. Sports-related correlates of disordered eating in aesthetic sports. Psychology of Sport and Exercise, Amsterdam, v.44, n.3, p.315-21, 2011.

LOHMAN, T.G. The use of skinfolds to estimate body fatness on children and youth. Journal Physical Education Recreation and Dance, Reston, v.58, n.1, p.98-103, 1987.

MACHADO, D.R.L.; BONFIM, M.R.; COSTA, R.T. Pico de velocidade de crescimento como alternativa para classificação maturacional associada ao desempenho motor. Revista Brasileira de Cineantropometria e Desempenho Humano, Florianópolis, v.11, n.1, p.14-21, 2009.

MALINA, R.; BOUCHARD, C.; BAR-OR, O. Crescimento, maturação e atividade física. São Paulo, Phorte: 2009. MARTINS, C.R.; PELEGRINI, A.; MATHEUS, S.C.; PETROSKI, E.L. Insatisfação com a imagem corporal e a relação com estado nutricional, adiposidade corporal e sintomas de anorexia e bulimia em adolescentes. Revista de Psiquiatria do Rio Grande do Sul, Porto Alegre, v.32, n.1, p.19-23, 2010. 
FORTES, L.S. et al.

MIRWALD, R.L.; BAXTER JONES, A.D.G.; BAILEY, D.A.; BEUNEN, G.P. An assessment of maturity from anthropometric measurements. Medicine and Sciense in Sport and Exercise, Madison, v.34, n.4, p.689-94, 2002.

PERINI, T.A.; VIEIRA, R.S.; VIGÁRIO; P.S.; OLIVEIRA, G.L.; ORNELLAS, J.S.; OILVEIRA, F.P. Transtorno do comportamento alimentar em atletas de elite de nado sincronizado. Revista Brasileira de Medicina do Esporte, São Paulo, v.15, n.1, p.54-7, 2009.

PETROSKI, E.L.; PELEGRINI, A.; GLANER, M.F. Insatisfação corporal em adolescentes rurais e urbanos. Revista Motricidade, Vila Real, v.5, n.4, p.13-25, 2009.

ROCHA, H.P.A.; BARTHOLO, T.L.; MELO, L.B.S.; SOARES, A.J.G. Jovens esportistas: profissionalização no futebol e a formação na escola. Motriz, Rio Claro, v.17, n.2, p.252-63, 2011.

SCHAAL, K.; TAFFLET, M.; NASSIF, H.; THIBAULT, V.; PICHARD, C.; ALCOTTE, M.; GUILLET, T.; BERTHELOT, G.; SIMON, S.; TOUSSAINT, J. Psychological balance in high level athletes: gender-based differences and sportspecific patterns. PLoS One, San Francisco, v.6, n.5, p.e19007, 2011. Disponível em: <http://www.insep.fr/FR/activites/ Recherchemedicales/publicationsscientifiques/Documents/Suivi\%20Psy\%20\%20Schaal\%20\%20PLoS\%20ONE\%20 -\%20copie.pdf>.

SCHERER, F.C.; MARTINS, C.R.; PELEGRINI, A.; MATHEUS, S.C.; PETROSKI, E.L. Imagem corporal em adolescentes: associação com a maturação sexual e sintomas de transtornos alimentares. Jornal Brasileiro de Psiquiatria, Rio de Janeiro, v.59, v.3, p.198-202, 2010.

SLAUGHTER, M.H.; LOHMAN, T.G.; BOILEAU, R.; HOSWILL, C.A.; STILLMAN, R.J.; YANLOAN, M.D. Skinfold equations for estimation of body fatness in children and youth. Human Biology, Detroit, v.60, n.3, p.709-23, 1988. TORSTVEIT, M.K.; SUNDGOT-BORGEN, J. The female athlete triad exists in both elite athletes and controls. Medicine and Science Sports and Exercise, Madison, v.37, n.1, p.1449-59, 2005.

VIEIRA, J.L.L.; VIEIRA, L.F.; AMORIM, H.Z.; ROCHA, P.G.M. Distúrbios de atitudes alimentares e sua relação com o crescimento físico de atletas paranaenses de Ginástica Rítmica. Motriz, Rio Claro, v.15, n.3, p.552-61, 2009.

WORLD HEALTH ORGANIZATION (WHO). Development of a WHO growth reference for school-aged children and adolescents. Bulletin of the World Health Organization, Geneve, v.85, n.9, p.660-7, 2007

ENDEREÇO

Leonardo de Sousa Fortes

R. Guaçui, 525/202

36025-190 - Juiz de Fora - MG - BRASIL e-mail: leodesousafortes@hotmail.com
Recebido para publicação: 03/ 10/2011

Revisado: 23/05/2012

Aceito: 25/ 05/2012 